\title{
ROTATION AND STABILITY OF THE TOROIDAL MAGNETIC FIELD IN STELLAR RADIATION ZONES
}

\author{
Alfio BonanNo ${ }^{1,2}$ AND VAdIM URPIN ${ }^{1,3,4}$ \\ ${ }^{1}$ INAF, Osservatorio Astrofisico di Catania, Via S.Sofia 78, I-95123 Catania, Italy; alfio.bonanno@inaf.it, vadim.urpin@uv.es \\ 2 INFN, Sezione di Catania, Via S.Sofia 72, I-95123 Catania, Italy \\ 3 A. F. Ioffe Institute of Physics and Technology, 194021 St. Petersburg, Russia \\ ${ }^{4}$ Isaac Newton Institute of Chile, Branch in St. Petersburg, 194021 St. Petersburg, Russia \\ Received 2012 September 6; accepted 2013 January 24; published 2013 March 6
}

\begin{abstract}
The stability of the magnetic field in radiation zones is of crucial importance for mixing and angular momentum transport in the stellar interior. We consider the stability properties of stars containing a predominant toroidal field in spherical geometry by means of a linear stability in the Boussinesq approximation taking into account the effect of thermal conductivity. We calculate the growth rate of instability and analyze in detail the effects of stable stratification and heat transport. We argue that the stabilizing influence of gravity can never entirely suppress the instability caused by electric currents in radiation zones. However, the stable stratification can essentially decrease the growth rate of instability.
\end{abstract}

Key words: instabilities - magnetohydrodynamics (MHD) - stars: magnetic field - Sun: interior

\section{INTRODUCTION}

The problem of the existence of a global magnetic field in the radiation zone of stars is still a topic of debate. Observations provide only upper limits on the strength of the magnetic field in the radiation zone of the Sun (see, e.g., Godier \& Rozelot 2000; Antia et al. 2000). For other stars estimates are much less certain and only theoretical upper limits can be derived. The possible origin of the field is also unclear. Perhaps, relic magnetic fields acquired by the star at the early stage of evolution can exist there. This type of field could have formed, for instance, because of a very weak differential rotation, which could have stretched the lines of a weak primordial seed field into a dominant toroidal field. Therefore, configurations with a predominantly toroidal field seem to be the most plausible in radiation zones. Generally the toroidal configurations can be present in different types of stars. For instance, they are typical for the liquid cores of neutron stars (Bonanno et al. 2005, 2006) where a large-scale toroidal magnetic field can be accompanied by small-scale magnetic structures (Urpin \& Gil 2004).

Toroidal magnetic configurations are subject to various instabilities which can shorten drastically the lifetime of the magnetic field. Many of these instabilities like the magnetic buoyancy instability (see, e.g., Gilman 1970; Acheson 1978) or the magnetorotational instability (Velikhov 1959; Balbus 1995) are well studied. However, in all probability, the most efficient instabilities are caused by electric currents maintaining the basic magnetic configuration, so that the evolution of the magnetic field is determined mainly by this type of instability (Spruit 1999). The latter are well studied in the context of laboratory fusion research (see, e.g., Goedbloed \& Poedts 2004) and, for this reason, in astrophysical conditions most of the present studies are in cylindrical geometry. In this case the stability properties of a pure toroidal field $B_{\varphi}$ can be characterized by the parameter $\alpha=d \ln B_{\varphi}(s) / d \ln s$, where $s$ is the cylindrical radius. The field is unstable to axisymmetric perturbations if $\alpha>1$ and to non-axisymmetric perturbations if $\alpha>-1 / 2$ (Tayler 1973; Markey \& Tayler 1973; Tayler 1980). Numerical modeling by Braithwaite (2006) confirmed that the toroidal field with $B_{\varphi} \propto s$ or $\propto s^{2}$ is unstable to the $m=1$ mode as was predicted by Tayler (1973) for cylindrical configurations. The current-driven instability might have, however, a number of characteristic features if both toroidal and axial field components are presented (Bonanno \& Urpin 2008a, 2008b). For instance, non-axisymmetric disturbances with large azimuthal wavenumbers $m$ turn out to be most rapidly growing in such configurations. Unstable disturbances exhibit a resonant character, i.e., the wave vector $\boldsymbol{k}=(\mathrm{m} / \mathrm{s}) \boldsymbol{e}_{\varphi}+k_{z} \boldsymbol{e}_{z}\left(k_{z}\right.$ is the wavevector in the axial direction) approximately satisfies the condition of magnetic resonance, $\boldsymbol{B} \cdot \boldsymbol{k}=0$. The length scale of this instability depends on the ratio of axial and azimuthal field components and it can be very short. This instability can play an important role, for instance, in various types of astrophysical jets (Bonanno \& Urpin 2011a, 2011b). The nonlinear stage of the Tayler instability was studied by Bonanno et al. (2012) who argued that symmetry-breaking can give rise to a saturated state with non-zero helicity even if the initial state has zero helicity. This conclusion is important for a possible dynamo action caused by the Tayler instability.

The stability of the spherical magnetic configurations is a much more complex issue and is qualitatively different from the cylindrical case. Therefore, some results obtained, for instance, for a toroidal configuration with $B_{\varphi}$ dependent on the cylindrical radius alone (see Spruit 1999; Zhang et al. 2003) do not apply to more realistic magnetic fields. Moreover, cylindrical geometry does not allow a detailed study of the effects of stratification and rotation which are of great importance in the radiation zone. It is widely believed that both these factors provide a stabilizing influence on the Tayler instability and can even suppress it entirely. Recently, Bonanno \& Urpin (2012) have considered the stability of the toroidal field in radiation zones taking into account stratification and thermal conductivity. The authors argued that the stabilizing influence of gravity and heat transport can never suppress entirely the current-driven instability but can only decrease its growth rate. The effect of rotation can also be important in stellar radiation zones, particularly, in rapidly rotating stars. The stability of azimuthal fields near the rotation axis has been studied by Spruit (1999). The author estimated the growth rate of instability and found that it can be of the order of $\sim \omega_{A}\left(\omega_{A} / \Omega\right)$ in the case of $\Omega \gg \omega_{A}$, where $\omega_{A}$ and $\Omega$ are the Alfvén frequency and the angular velocity of a star, respectively. However, this simple estimate applies only near the axis and is not valid in the main fraction of the volume of a radiation zone (see Mathis \& Zahn 2005). A qualitatively different result has been obtained for a rotational suppression 
by Braithwaite (2006). In his numerical modeling, the Tayler instability was suppressed if $\Omega$ is above a certain value of the order of $\omega_{A}$. Above this value a distinct oscillatory behavior sets in with marginal stability. The nature of oscillatory modes has not been analyzed. The stability of the toroidal field in a rotating radiation zone in the particular case $B_{\varphi} \propto s$ has been studied by Zahn et al. (2007). The authors found that a particular type of oscillatory mode should exist with the frequency $\sim \omega_{A}\left(\omega_{A} / \Omega\right)$ comparable to the growth rate estimated by Spruit (1999). These modes are stable in the non-dissipative limit but they can be unstable if, for example, radiative heat transport is taken into account. Stability of the toroidal field in rotating stars has been considered also by Kitchatinov (2008) and Kitchatinov \& Rüdiger (2008) who argued that the magnetic instability is determined by a threshold field strength at which the instability sets in. Estimating this threshold in the solar radiation zone, Kitchatinov \& Rüdiger (2008) impose the upper limit on the magnetic field in the radiation zone of the Sun $\approx 600 \mathrm{G}$.

In this paper we consider the combined influence of stratification, thermal conductivity, and rotation on the stability of the toroidal field in a stellar radiation zone. These factors are always presented in radiation zones and therefore it is of great importance to consider the stability under their combined influence. The paper is organized as follows. The basic equations and mathematical formulation of the problem are presented in Section 2. The results of the numerical calculations of the growth rate and frequency of the instability are discussed in Section 3. Section 4 contains a brief summary of the main results and the conclusions.

\section{BASIC EQUATIONS}

Let us consider the stability of an axisymmetric toroidal magnetic field neglecting viscosity and magnetic diffusivity. We work in spherical coordinates $(r, \theta, \varphi)$ with the unit vectors $\left(\boldsymbol{e}_{r}\right.$, $\left.\boldsymbol{e}_{\theta}, \boldsymbol{e}_{\varphi}\right)$. We assume that the radiation zone rotates rigidly with the angular velocity $\boldsymbol{\Omega}$ and that the toroidal field depends on $r$ and $\theta, B_{\varphi}=B_{\varphi}(r, \theta)$. We consider the stability using the limit of incompressible fluid. This limit applies if the gas pressure is greater than the magnetic pressure (see, e.g., Landau \& Lifshitz 1959). In this limit, the MHD equations read

$$
\begin{gathered}
\frac{\partial \boldsymbol{v}}{\partial t}+(\boldsymbol{v} \cdot \nabla) \boldsymbol{v}=-\frac{\nabla p}{\rho}+\boldsymbol{g}+\frac{1}{4 \pi \rho}(\nabla \times \boldsymbol{B}) \times \boldsymbol{B}, \\
\frac{\partial \boldsymbol{B}}{\partial t}-\nabla \times(\boldsymbol{v} \times \boldsymbol{B})=0, \\
\nabla \cdot \boldsymbol{v}=0, \quad \nabla \cdot \boldsymbol{B}=0,
\end{gathered}
$$

where $\boldsymbol{g}$ is gravity. The equation of thermal balance reads in the Boussinesq approximation

$$
\frac{\partial T}{\partial t}+v \cdot\left(\nabla T-\nabla_{\mathrm{ad}} T\right)=\nabla \cdot(\kappa \nabla T),
$$

where $\kappa$ is the thermal diffusivity and $\nabla_{\mathrm{ad}} T$ is the adiabatic temperature gradient.

In the basic (unperturbed) state, the gas is assumed to be in hydrostatic equilibrium, then

$$
\frac{\nabla p}{\rho}=\boldsymbol{g}+\frac{1}{4 \pi \rho}(\nabla \times \boldsymbol{B}) \times \boldsymbol{B}+\boldsymbol{e}_{s} \Omega^{2} r \sin \theta,
$$

where $\boldsymbol{e}_{s}$ is the unit vector in the cylindrical radial direction. The rotational energy is assumed to be much smaller than the gravitational one, $g \gg r \Omega^{2}$. Since the magnetic energy is subthermal, $\boldsymbol{g}$ is approximately radial in our basic state.

We consider a linear stability. Linearizing Equations (1)-(4), we take into account that small perturbations of the density and temperature in the Boussinesq approximation are related by $\rho_{1} / \rho=-\beta\left(T_{1} / T\right)$, where $\beta$ is the thermal expansion coefficient. For small perturbations, we use a local approximation in the $\theta$-direction and assume that their dependence on $\theta$ is proportional to $\exp (-i l \theta)=\exp \left(-k_{\theta} r \theta\right)$, where $l \gg 1$ and $k_{\theta}=l / r$ are the longitudinal wavenumber and wavevector, respectively. Since the basic state is stationary and axisymmetric, the dependence of perturbations on $t$ and $\varphi$ can be taken in the exponential form as well. Then, perturbations are proportional to $\exp (\sigma t-i l \theta-i m \varphi)$, where $m$ is the azimuthal wavenumber. The dependence on $r$ should be determined from Equations (1)-(4). For the sake of simplicity, we assume that unperturbed $\rho$ and $T$ are approximately homogeneous in the radiation zone. This assumption does not change the main conclusions qualitatively but simplifies substantially calculations. Eliminating all variables in the linearized Equations (1)-(4) in favor of the perturbations of the radial velocity $v_{1 r}$ and temperature $T_{1}$, we obtain with the accuracy in terms of the lowest order in $\left(k_{\theta} r\right)^{-1}$ the set of two coupled equations:

$$
\begin{aligned}
\left(\sigma_{1}^{2}+\right. & \left.\omega_{A}^{2}+D \Omega_{i}^{2}\right) v_{1 r}^{\prime \prime}+\left(\frac{4}{r} \sigma_{1}^{2}+\frac{2}{H} \omega_{A}^{2}\right) v_{1 r}^{\prime} \\
+ & {\left[\frac{2}{r^{2}} \sigma_{1}^{2}-k_{\perp}^{2}\left(\sigma_{1}^{2}+\omega_{A}^{2}\right)-D \Omega_{e}^{2} k_{\theta}^{2}\right.} \\
& +\frac{2}{r} \omega_{A}^{2}\left(\frac{1}{H} \frac{k_{\perp}^{2}}{k_{\varphi}^{2}}-\frac{2}{r} \frac{k_{\theta}^{2}}{k_{\varphi}^{2}} D\right)-i \sigma_{1} \Omega_{e} \\
& \left.\times\left(\frac{k_{\varphi}}{r}+4 D \frac{k_{\theta}^{2}}{r k_{\varphi}} \frac{\omega_{A}^{2}}{\sigma_{1}^{2}}\right)\right] v_{1 r}=-k_{\perp}^{2} \beta g \sigma_{1} \frac{T_{1}}{T}, \\
\frac{\kappa}{r^{2}} \frac{\partial}{\partial r} & {\left[r^{2} \frac{\partial}{\partial r}\left(\frac{T_{1}}{T}\right)\right]-\left(\sigma_{1}+\kappa k_{\perp}^{2}\right) \frac{T_{1}}{T}=\frac{\omega_{\mathrm{BV}}^{2}}{\beta g} v_{1 r}, }
\end{aligned}
$$

where the prime denotes a derivative with respect to $r$ and

$$
\begin{aligned}
\sigma_{1} & =\sigma-i m \Omega, \quad \omega_{A}^{2}=\frac{k_{\varphi}^{2} B_{\varphi}^{2}}{4 \pi \rho}, \\
\omega_{\mathrm{BV}}^{2} & =-\frac{g \beta}{T}\left(\nabla_{\mathrm{ad}} T-\nabla T\right)_{r}, \quad D=\frac{\sigma_{1}^{2}}{\sigma_{1}^{2}+\omega_{A}^{2}}, \\
\Omega_{i} & =2 \Omega \cos \theta, \quad \Omega_{e}=2 \Omega \sin \theta, \quad k_{\perp}^{2}=k_{\theta}^{2}+k_{\varphi}^{2}, \\
k_{\varphi} & =\frac{m}{r \sin \theta}, \quad \frac{1}{H}=\frac{\partial}{\partial r} \ln \left(r B_{\varphi}\right) .
\end{aligned}
$$

If $\Omega=0$, this set of equations transforms into the set derived by Bonanno \& Urpin (2012) for non-rotating radiation zones. The toroidal field does not enter the equation of thermal balance (7) because the thermal transport is not influenced by the field. On the contrary, $B_{\varphi}(r, \theta)$ plays an important role in Equation (6) since $\omega_{A}$ is proportional to it. Therefore, the growth rate is determined by both the latitudinal and radial profiles of $B_{\varphi}(r, \theta)$. Note that the toroidal field enters Equation (6) as a parameter but the latitudinal derivative of $B_{\varphi}$ does not enter. Often, however, stability criteria for the Tayler instability in the spherical geometry are formulated in terms of the latitudinal profile of $B_{\varphi}$ and its $\theta$-derivative (see, e.g., Goossens et al. 1981). Although our approach is suited to perform calculations 
of the growth rate, it can also be used to study the stability condition. This condition should contain both the toroidal field and its derivative with respect to a latitude. Indeed, the condition of stability reads in our approach $\max \Gamma(\theta)<0$ (at fixed values of other parameters). The value of $\theta^{*}$ where $\Gamma$ is maximal is determined by the equation $\partial \Gamma(\theta) / \partial \theta=0$. This condition depends on the latitudinal profile and, as a result, the criterion of instability should depend on the $\theta$-derivative of $B_{\varphi}$ although Equations (6) and (7) for $\Gamma$ do not depend. The present paper, however, basically addresses calculations of the growth rate, and the instability conditions will not be considered.

Some general stability properties can be derived directly from Equations (6) and (7). Consider perturbations with a very short radial wavelength for which one can use a local approximation in the radial direction, such as $v_{1 r} \propto \exp \left(-i k_{r} r\right)$, where $k_{r}$ is the radial wavevector. If $k_{r} \gg \max \left(k_{\theta}, k_{\varphi}\right)$, then Equations (6) and (7) can be reduced with the accuracy in terms of the lowest order in $\left(k_{r} r\right)^{-1}$ to the following set of algebraic equations:

$-\left(\sigma_{1}+\kappa k^{2}\right) \frac{T_{1}}{T}=\frac{\omega_{\mathrm{BV}}^{2}}{\beta g} v_{1 r}, \quad k_{r}^{2}\left(\sigma_{1}^{2}+\omega_{A}^{2}+D \Omega_{i}^{2}\right) v_{1 r}=k_{\perp}^{2} \beta g \sigma \frac{T_{1}}{T}$,

where $k^{2}=k_{r}^{2}+k_{\perp}^{2}$. The corresponding dispersion relation reads

$$
\begin{gathered}
\sigma_{1}^{5}+\kappa k^{2} \sigma_{1}^{4}+\left(2 \omega_{A}^{2}+\Omega_{i}^{2}+\frac{k_{\perp}^{2}}{k^{2}} \omega_{\mathrm{BV}}^{2}\right) \sigma_{1}^{3}+\kappa k^{2}\left(2 \omega_{A}^{2}+\Omega_{i}^{2}\right) \sigma_{1}^{2} \\
+\omega_{A}^{2}\left(\omega_{A}^{2}+\frac{k_{\perp}^{2}}{k^{2}} \omega_{\mathrm{BV}}^{2}\right) \sigma_{1}+\kappa k^{2} \omega_{A}^{4}=0
\end{gathered}
$$

The condition that at least one of the roots has a positive real part (unstable mode) is determined by the Routh criterion (see, e.g., Aleksandrov et al. 1963). For a particular case of an equation of the fifth order, these criteria have been derived by Urpin $\&$ Rüdiger (2005). These criteria yield the only non-trivial condition of instability $\omega_{\mathrm{BV}}^{2}<0$, which is not satisfied in the radiation zone by definition. Therefore, modes with short radial wavelength are always stable to the current-driven instability. This conclusion was first obtained by Bonanno \& Urpin (2012) for non-rotating radiative zones and, here, we generalize the result for the case of rotating stars.

\section{NUMERICAL RESULTS}

We assume that the inner and outer boundaries of the radiation zone are located at $r=R_{i}$ and $r=R$, respectively. Introducing the dimensionless radius $x=r / R$, we obtain for the boundaries $x=x_{i}=R_{i} / R$ and $x=1$. We choose the internal radius of the radiation zone, $x_{i}$, to be small but finite, ranging from $x_{i}=0.1$ to $x_{i}=0.4$ in all the simulations. We did not find qualitative difference from $x_{i}=0.1$ to $x_{i}=0.4$, but the numerical stability of the system was improved in the latter case. All the figures presented in the paper are therefore obtained for $x_{i}=0.4$.

The toroidal field can be conveniently represented as

$$
B_{\varphi}=B_{0} \psi(x) \sin \theta, \quad \psi(x)=\left(x / x_{i}\right)^{\alpha},
$$

where $B_{0}$ is the field strength at $x=x_{i}$ at the equator. Since the dependence of $B_{\varphi}$ on $r$ is uncertain in the radiation zone, we consider different possibilities, varying $\alpha$. The models with $\alpha>0$ where the field reaches its maximum at the outer boundary can mimic, for example, the radiative interior of a star with a convective envelope. In this case, the bottom of the convection zone is the location of the toroidal field generated by a dynamo action which can penetrate into the radiation zone.
Introducing the dimensionless quantities as

$$
\Gamma=\frac{\sigma_{1}}{\omega_{A 0}}, \quad \delta^{2}=\frac{\omega_{\mathrm{BV}}^{2}}{\omega_{A 0}^{2}}, \quad \varepsilon=\frac{\omega_{T}}{\omega_{A 0}}, \quad \eta=\frac{2 \Omega}{\omega_{A 0},}
$$

where $\omega_{A 0}^{2}=B_{0}^{2} / 4 \pi \rho R^{2}$ and $\omega_{T}=\kappa / R^{2}$, we can transform Equations (6) and (7) to the dimensionless form. They read

$$
\begin{gathered}
\left(\Gamma^{2}+\frac{\psi^{2}}{x^{2}}+E \eta^{2} \cos ^{2} \theta\right) \frac{d^{2} v_{1 r}}{d x^{2}}+\frac{1}{x}\left(4 \Gamma^{2}+\frac{2}{x} N \psi^{2}\right) \frac{d v_{1 r}}{d x} \\
+\frac{v_{1 r}}{x^{2}}\left[2 \Gamma^{2}-q^{2}\left(\Gamma^{2}+\frac{\psi^{2}}{x^{2}}\right)-E \eta^{2} l^{2} \sin ^{2} \theta\right. \\
-i \eta \Gamma\left(m+\frac{4 E \psi^{2}}{x^{2} \Gamma^{2}} \frac{l^{2} \sin ^{2} \theta}{m}\right) \\
\left.+\frac{2 \psi^{2}}{m^{2} x}\left(q^{2} N \sin ^{2} \theta-\frac{2}{x} E l^{2} \sin ^{2} \theta\right)\right]=-\frac{\Gamma}{x^{2}} q^{2} u \\
\frac{\varepsilon}{x^{2}} \frac{d}{d x}\left(x^{2} \frac{d u}{d x}\right)-\left(\Gamma+\frac{\varepsilon}{x^{2}} q^{2}\right) u=\delta^{2} v_{1 r}
\end{gathered}
$$

where

$$
\begin{aligned}
u & =\frac{\beta g}{\omega_{A 0}} \frac{T_{1}}{T}, \quad E=\frac{\Gamma^{2}}{\Gamma^{2}+\psi^{2} / x^{2}}, \quad N=\frac{R}{H}=\frac{1}{x}+\frac{1}{\psi} \frac{d \psi}{d x}, \\
q^{2} & =l^{2}+\frac{m^{2}}{\sin ^{2} \theta} .
\end{aligned}
$$

The parameters $\delta^{2}$ and $\varepsilon$ characterize the effect of stratification and thermal conductivity, respectively, and $\eta$ describes a stabilizing influence of rotation. Equations (13) and (14) with the corresponding boundary conditions describe the stability problem as a nonlinear eigenvalue problem. Fortunately, the qualitative features of the problem are not particularly sensitive to the choice of boundary conditions. That is why we choose the simplest conditions and assume that $v_{1 r}=T_{1}=0$ at $r=R_{i}$ and $r=R$. Note that the parameter $\delta$ is large in radiation zones but, most likely, $\varepsilon$ is relatively small if the magnetic field is not very weak. In calculations, we suppose $\delta$ and $\varepsilon$ to be constant through the radiation zone. As it was already mentioned the coefficients of Equations (6) and (7) (and the corresponding dimensionless equations) depend on $\theta$ and this property leads to a latitudinal dependent $\Gamma=\Gamma(\theta)$. In the present paper, only the simplest latitudinal profile $B_{\varphi} \propto \sin \theta$ is considered because this dependence is often used in modeling the toroidal field in stars (see, e.g., Kitchatinov \& Rüdiger 2008). For other dependences of $B_{\varphi}$ on $\theta$, the results can differ quantitatively but the main qualitative features are same. Generally, Equations (13) and (14) are complex. It is more convenient to split all quantities into the real and imaginary parts and to solve numerically the set of four real equations that follows from Equations (13) and (14).

The stability properties in the spherical geometry can be qualitatively different from those in the cylindrical geometry (Bonanno \& Urpin 2012). To illustrate this point, we plot in a few figures the growth rate and frequency of the instability as a function of the rotational parameter $\eta$ for different polar angles, $\theta$, and for $\alpha=2$. In Figure 1, the growth rate is shown at the equator. It turns out that a combined influence of stratification and rotation provides some stabilizing effect on the instability. The growth rate is maximal for a nonrotating star with neutral stratification $\left(\delta^{2}=0\right)$. At small $\eta$ 

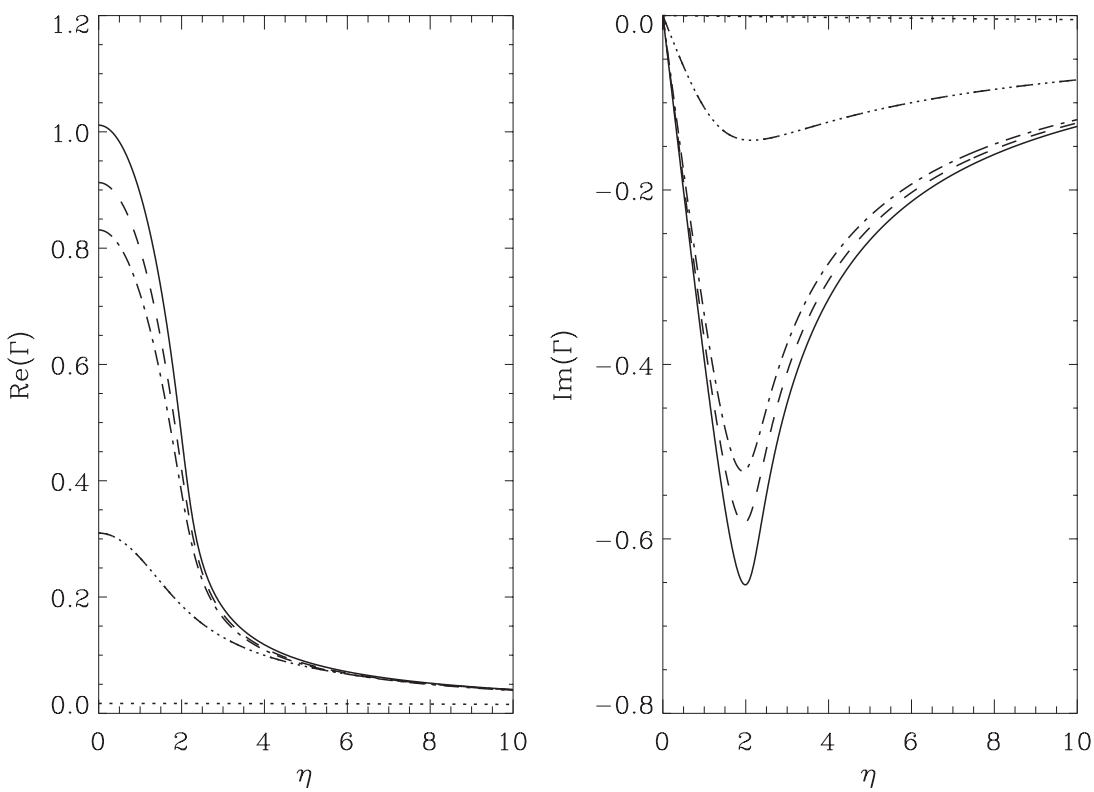

Figure 1. Growth rate (left panel) and frequency (right panel) of the Tayler's modes at the equator as functions of the rotational parameter $\eta$ for $\alpha=2, \varepsilon=0.01$ and for $\delta^{2}=0$ (solid), 4 (dashed), 8 (dash-dotted), 100 (dash-dot-dotted), and 1000 (dotted). The azimuthal and longitudinal wavenumbers are $m=1$ and $l=10$, respectively.
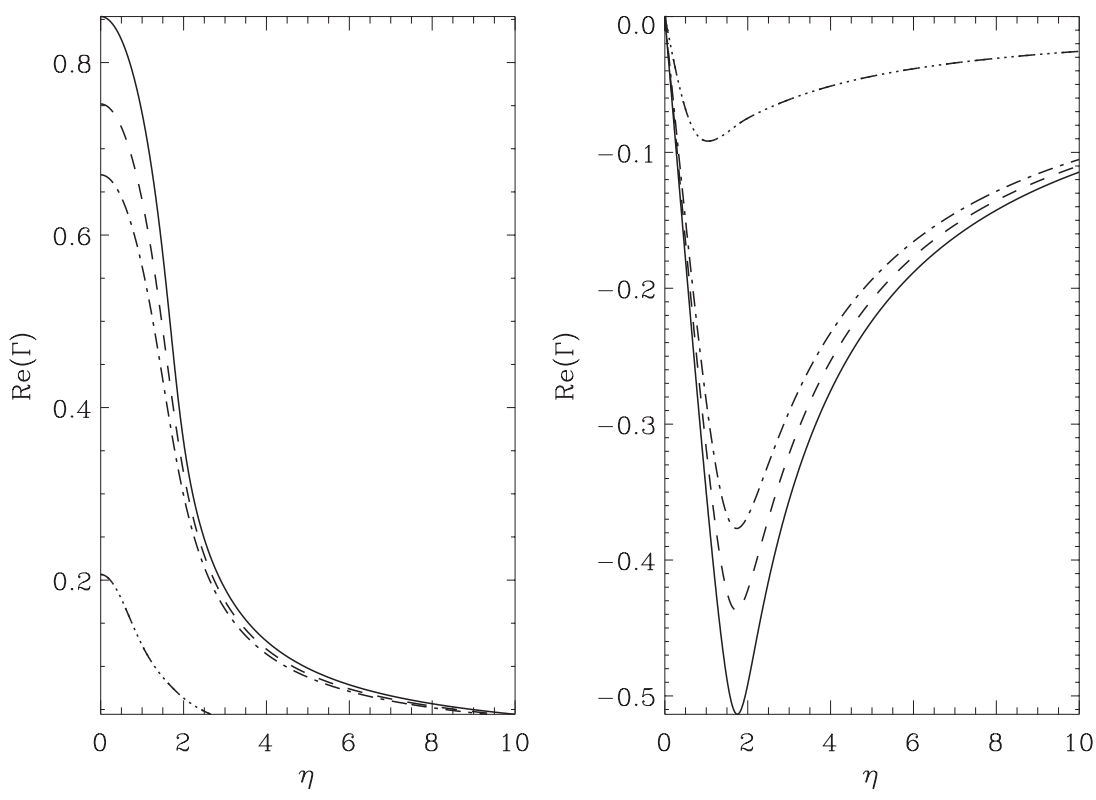

Figure 2. Same as in Figure 1 but for $\theta=60^{\circ}$.

and $\delta^{2} \ll 1$, the growth rate is of the order of $\omega_{A 0}$, but it clearly shows some suppression for a faster rotation. The growth rate exhibits a qualitatively same rotational suppression for the radiation zone with a higher stratification parameter $\delta$. Suppression becomes important already at relatively small values of $\eta \sim 1-2$. However, this suppression cannot stop the instability but it only reduces the growth rate. The growth rate decreases with an increase of $\eta$ approximately as $1 / \eta$ and it does not vanish even at very large $\eta$. A similar behavior in the case of ideal plasma was obtained by Spruit (1999) who considered the instability near the rotation axis in the case of the toroidal field dependent on the cylindrical radius alone, $B_{\varphi}=B_{\varphi}(s)$. Stratification leads to an additional decrease of the growth rate. At $\eta<1$, for example, the growth rate is by a factor of $\approx 3$ smaller for $\delta^{2}=100$ compared to $\delta^{2}=0$. At very large $\delta$, the growth rate decreases even more, but it does not reach 0 at any finite $\delta$. It turns out that, in the case of $\alpha=2$, the combined influence of stratification, rotation, and thermal conductivity can never stop the Tayler instability in the region near the equator but can only decrease the growth rate. Note that the Tayler's modes are oscillatory in contrast to the non-rotating case. The frequency is basically comparable to the growth rate and also decreases for faster rotation.

In Figure 2, the growth rate of instability is presented for the same model of the magnetic field with $\alpha=2$ but for another polar angle, $\theta=60^{\circ}$. Qualitatively, a behavior of the growth rate is the same as in the previous case. A combined influence of stratification and rotation provides a stabilizing effect on the instability. Like the case $\theta=90^{\circ}$, the growth rate at given $\delta$ is maximal in a non-rotating star and it decreases substantially 

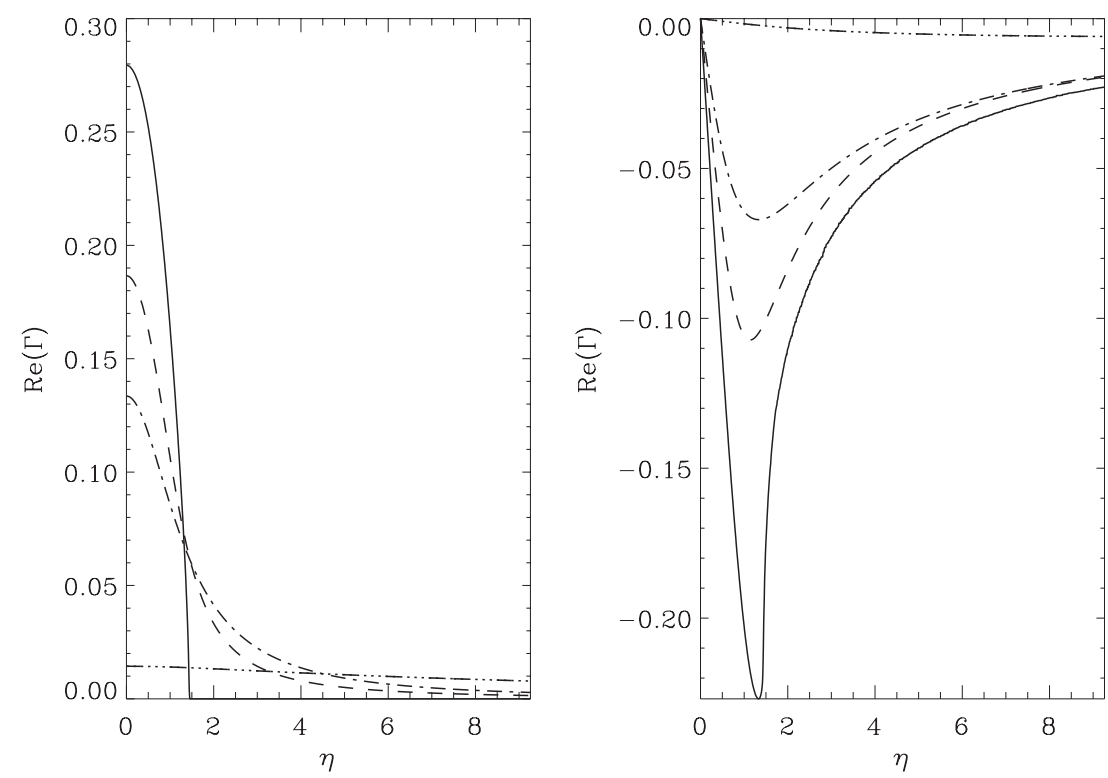

Figure 3. Same as in Figures 1 and 2 but for $\theta=30^{\circ}$.
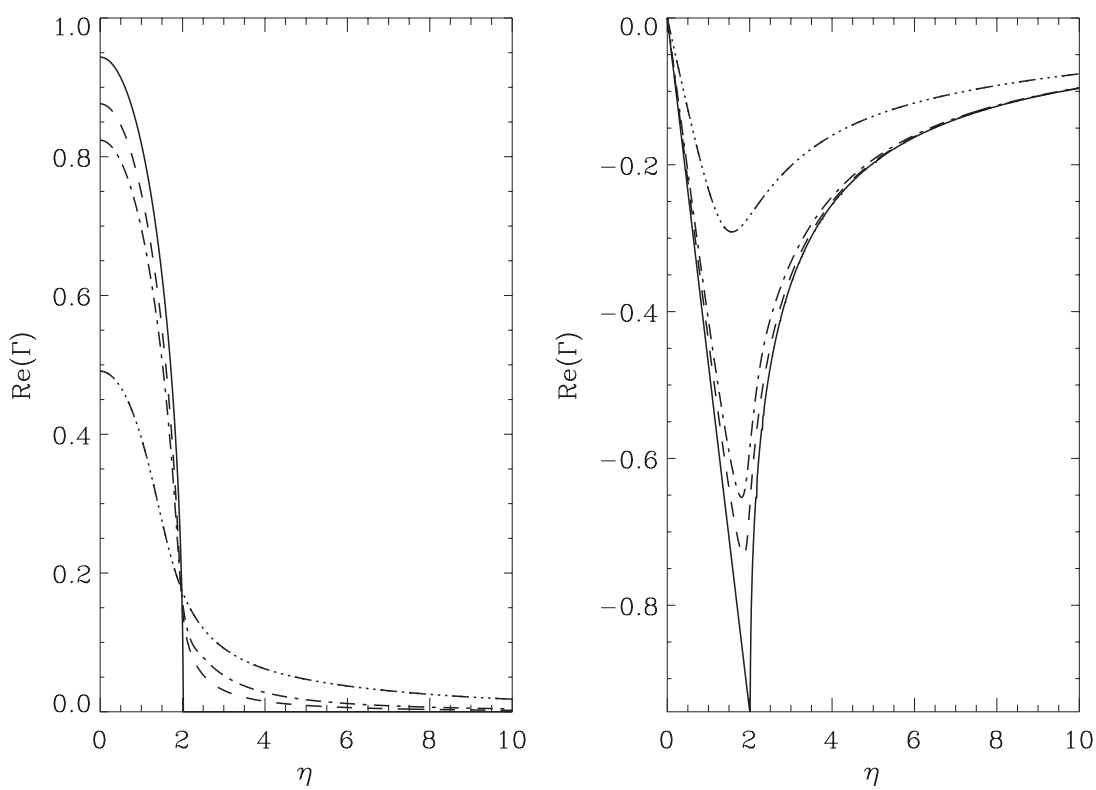

Figure 4. Growth rate (left panel) and frequency (right panel) of the Tayler's modes at the equator as functions of the rotational parameter $\eta$ for $\alpha=1, \varepsilon=0.01$ and for $\delta^{2}=0$ (solid), 4 (dashed), 8 (dash-dotted), 100 (dash-dot-dotted), and 1000 (dotted). The azimuthal and longitudinal wavenumbers are $m=1$ and $l=10$, respectively.

for a faster rotation. Suppression of the growth rate becomes important at relatively small values of $\eta \sim 1-2$ but, however, this suppression cannot stop the instability and only reduces the growth rate. Like the previous case, for any given $\delta$, the growth rate decreases with an increase of $\eta$ approximately $\propto 1 / \eta$. Stratification also leads to a decrease of the growth rate. For example, at small $\eta$, the growth rate is $\approx 4$ smaller for $\delta^{2}=100$ than for $\delta^{2}=0$. At very large $\delta$ (but small $\eta$ ), the growth rate decreases even more but it does not vanish at any large $\delta$ (see also Bonanno \& Urpin 2012). Unstable modes are oscillatory in contrast to the non-rotating case and their frequency is comparable to the growth rate.

In Figure 3. we plot the growth rate and frequency of unstable modes for $\theta=30^{\circ}$. It seems that the stabilizing influence of rotation and stratification is stronger in the region close to the axis. A comparison of Figures 1 and 3 shows that the instability is most efficient at the equator and very weak close to the axis. Note that the instability exhibits the same behavior also in the non-rotating case (Bonanno \& Urpin 2012). This result is at variance with the widely accepted opinion that the toroidal magnetic field in stars is always unstable around the rotation axis (see, e.g., Spruit 1999). This opinion is based on a seeming similarity of the spherical magnetic configuration near the axis and the axisymmetric cylindrical configuration. However, this analogy is generally incorrect because, in spherical geometry, the toroidal field near the axis depends also on the coordinate along the axis not only on the distance from it. In contrast to the instability near the equator, the instability at $\theta=30^{\circ}$ can be characterized by the threshold, $\eta_{\mathrm{cr}}$, if the stratification parameter $\delta$ is small. The instability occurs if $\eta<\eta_{\mathrm{cr}}$ and it is entirely suppressed if $\eta>\eta_{\mathrm{cr}}$ for small $\delta$. The threshold is not high and corresponds to $\eta_{\mathrm{cr}} \sim 1.5$ if $\delta=0$. Therefore, even a relatively 

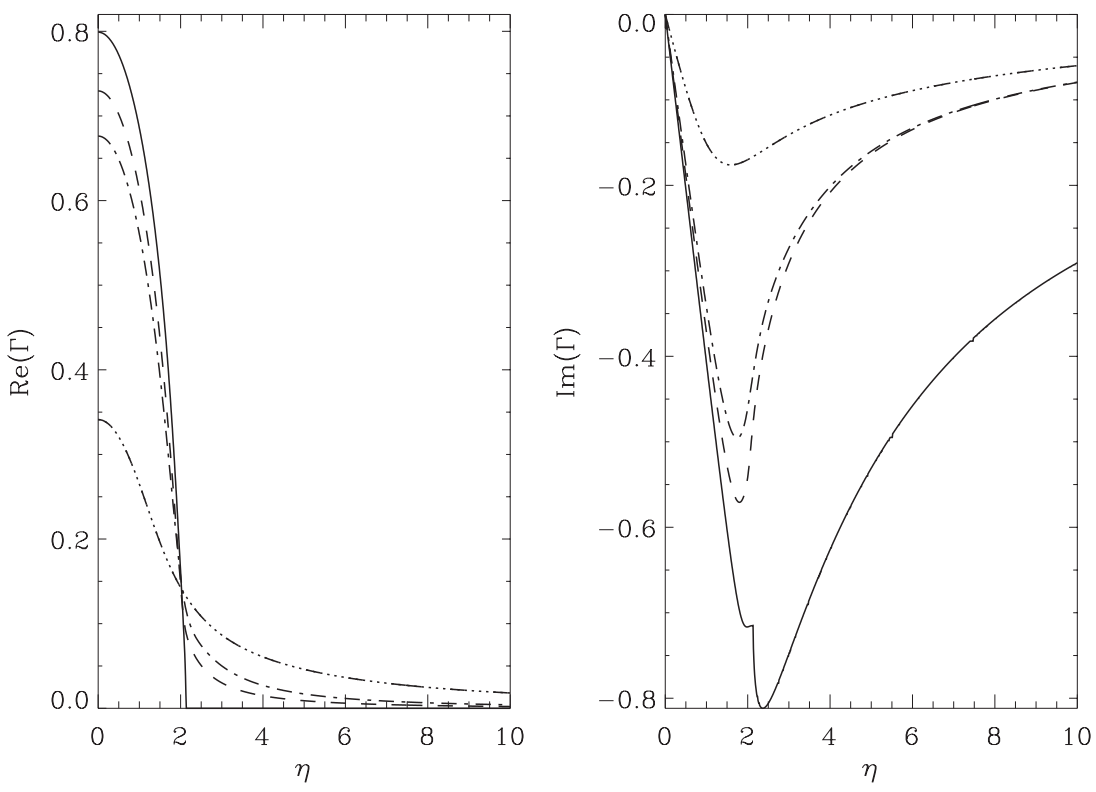

Figure 5. Same as in Figure 4 but for $\theta=60^{\circ}$.
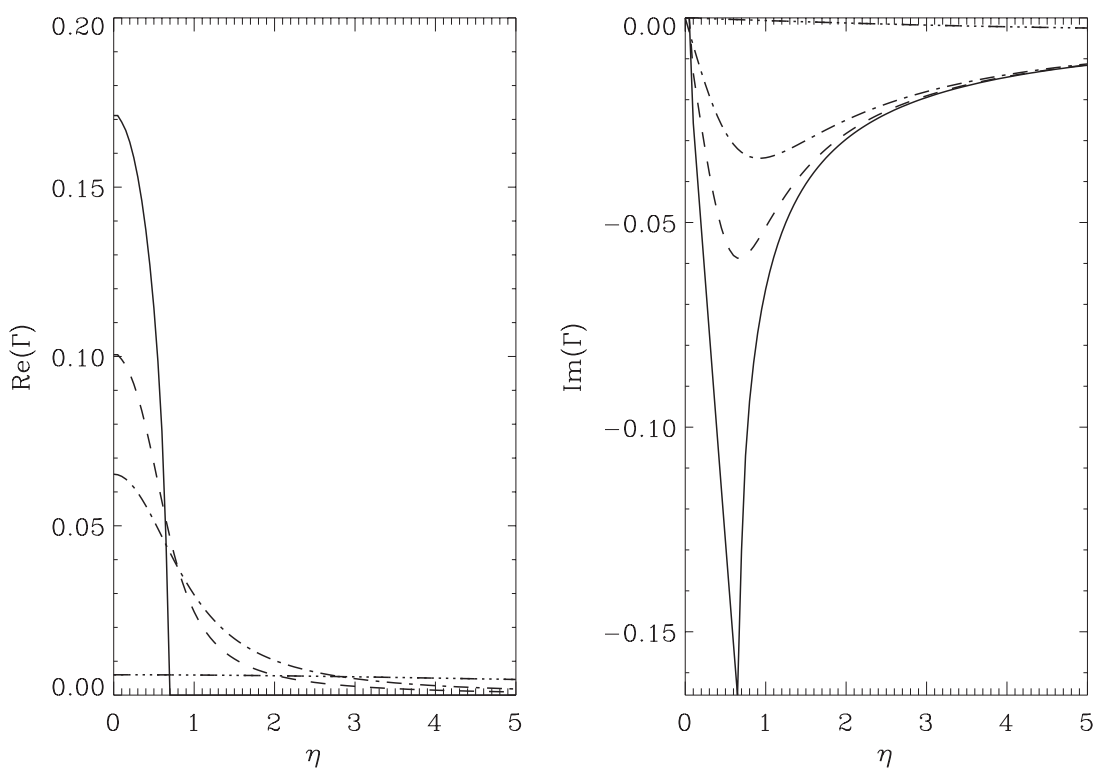

Figure 6. Same as in Figure 4 but for $\theta=33^{\circ}$.

slow rotation with $\eta>1.5$ can stop the instability in some region around the axis if stratification is close to the neutral one. Note that the Tayler modes become oscillatory and marginally stable beyond the threshold: their growth rate is equal to zero but the frequency is always non-vanishing. The instability has no threshold if $\delta$ is not small. In this case, the growth rate does not vanish even at very large $\eta$ but it decreases $\propto 1 / \eta$.

The growth rate of the instability at the equator is shown in Figure 4 for the case $\alpha=1$. Qualitatively, the behavior is the same but the instability turns out to be a bit weaker in this case. At $\alpha=2$, a combined influence of stratification, rotation, and thermal conductivity can never suppress the Tayler instability in the region near the equator but can only decrease the growth rate. At variance with the previous case in the case $\alpha=1$ this influence is more pronounced and can even suppress the instability entirely if $\eta>2$ and $\delta \ll 1$. At larger $\delta$, the instability cannot be stopped neither by rotation nor by stratification but its growth rate decreases substantially. Like the previous case, the instability is oscillatory with the frequency comparable to the growth rate. The frequency reaches its maximum, $\operatorname{Im} \sigma \sim \omega_{A 0}$, at $\Omega \sim \omega_{A 0}$ and, then, decreases in more rapidly rotating stars as $1 / \eta \propto 1 / \Omega$.

In Figure 5, we plot $\Gamma$ as a function of $\eta$ in the case $\alpha=1$ and for $\theta=60^{\circ}$. Again, in contrast to the case $\alpha=2$, the instability can be suppressed completely at $\eta>2$ and a weak stratification, $\delta \ll 1$. However, if stratification is stronger, the instability can occur at large $\eta$ but with a substantially reduced growth rate. The unstable modes are always oscillatory and their frequency is comparable to (or a bit greater than) the growth rate. At small $\delta \ll 1$ and $\eta>2$, the modes are marginally stable $(\operatorname{Re} \Gamma \approx 0)$ but their frequency is non-vanishing.

Figure 6 shows $\Gamma$ versus $\eta$ for $\alpha=1$ and $\theta=33^{\circ}$. The growth rate of instability is substantially smaller than in the regions close to the equator (Figures 4 and 5). As already mentioned, this is a rather general behavior in spherical geometry: the instability becomes weaker near the pole and 

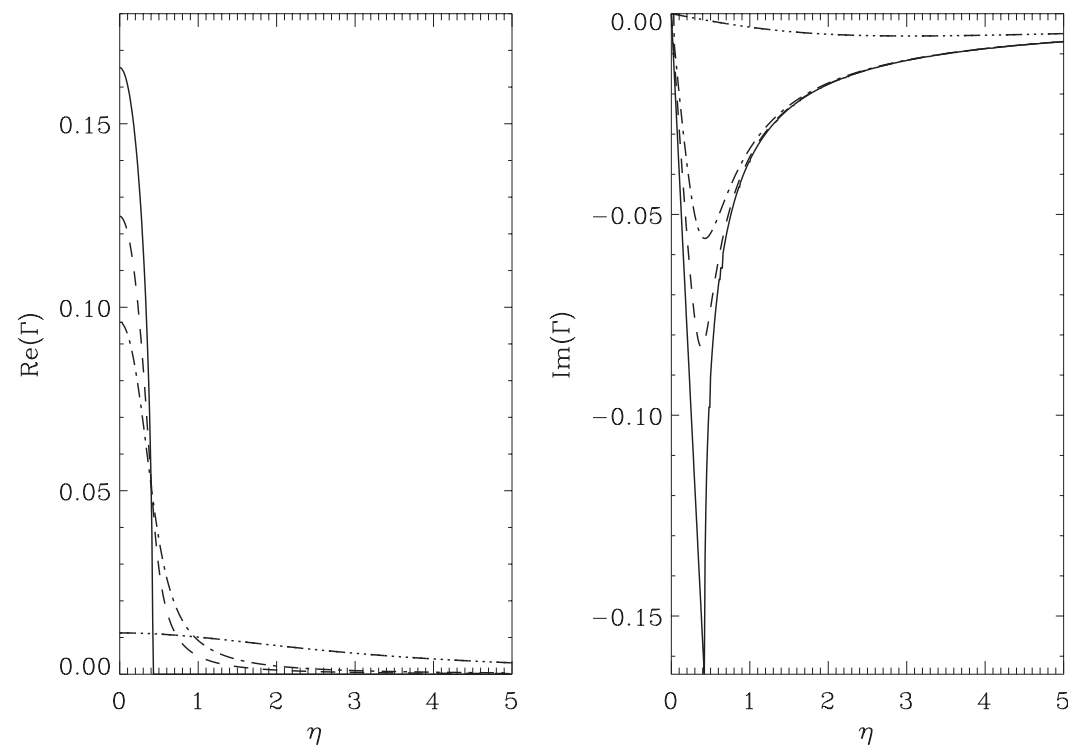

Figure 7. Growth rate (left panel) and frequency (right panel) of the Tayler's modes at the equator as functions of the rotational parameter $\eta$ for $\alpha=-0.4, \varepsilon=0.01$ and for $\delta^{2}=0$ (solid), 4 (dashed), 8 (dash-dotted), 100 (dash-dot-dotted), and 1000 (dotted). The azimuthal and longitudinal wavenumbers are $m=1$ and $l=10$, respectively.
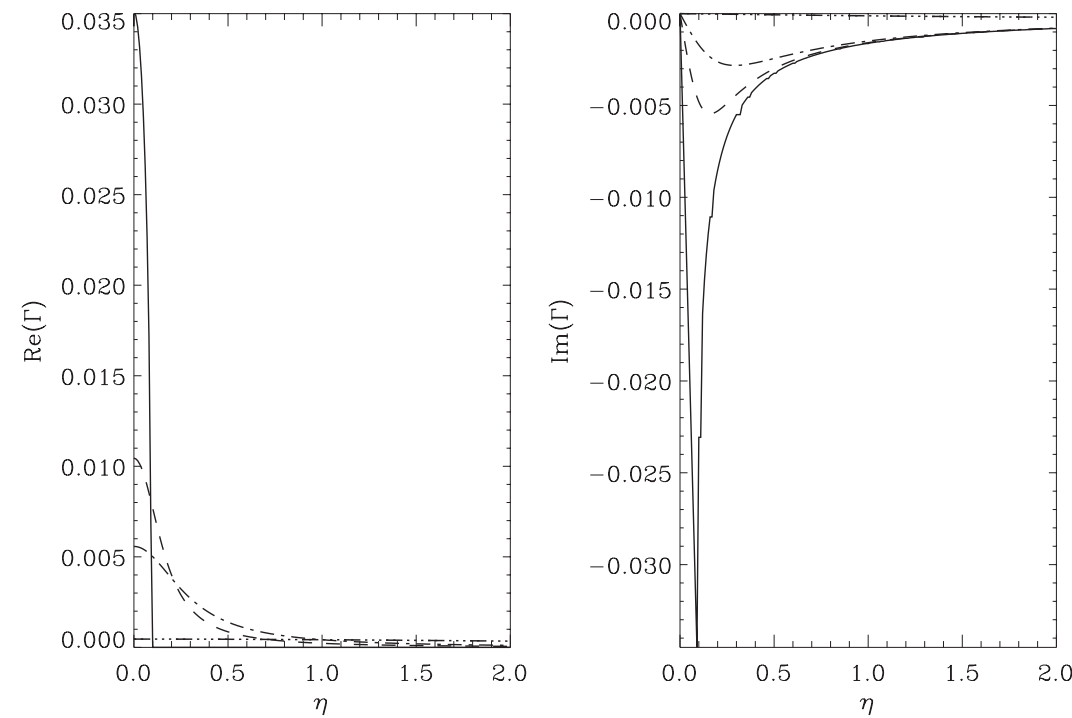

Figure 8. Same as in Figure 7 but for $\theta=80^{\circ}$.

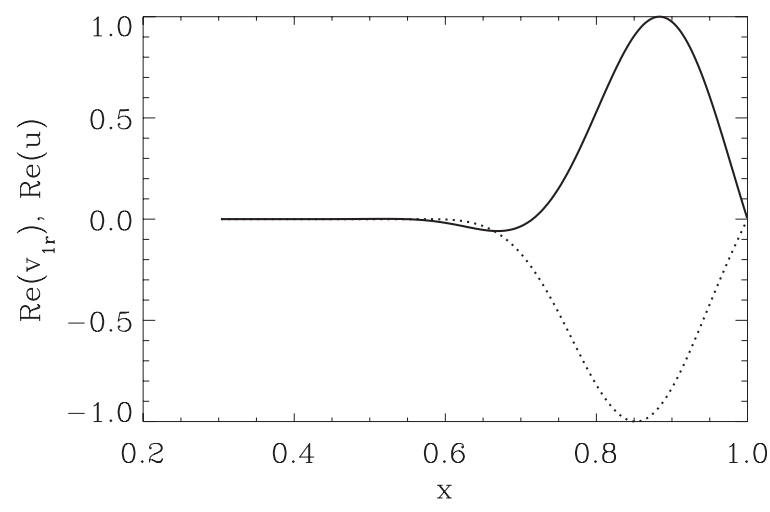

Figure 9. Radial profile of the real part of the eigenfunctions $v_{1 r}$ (solid) and $u$ (dashed) for $\alpha=2, \eta=2, q=12, \delta=4, \epsilon=0.02$, and $\theta=90^{\circ}$.

usually ceases at the axis. For example, the growth rate is approximately five times smaller at $\theta=33^{\circ}$ than at $\theta=60^{\circ}$ if $\eta \ll 1$ and $\delta=0$, and $\approx 10$ times smaller if $\delta^{2}=$ 8. As everywhere in the radiation zone, the instability is oscillatory with the frequency being of the order of the growth rate.

In Figures 7 and 8 we plot $\Gamma$ as a function of $\eta$ for $\alpha=-0.4$ and $\theta=90^{\circ}$ and $\theta=80^{\circ}$, respectively, the angles below $\theta=75^{\circ}$ being stable. As should now be clear, smaller values of $\alpha$ imply a weaker instability. We did not find any instability if $\alpha<-0.5$ and therefore we did not considered the behavior of the modes in the neighborhood of this critical value.

\section{CONCLUSION}

We have considered the stability of the toroidal magnetic field in stellar radiation zones taking into account the effects of rotation, stratification, and thermal conductivity. Our consideration is local in the $\theta$-direction and, as a result, we reduce the stability analysis to a nonlinear eigenvalue problem for a second-order differential equation in $r$. The eigenfunctions, corresponding to unstable modes, are usually localized in a neighborhood of the outer (if $\alpha>1$ ) or inner (if $\alpha<1$ ) boundary and are relatively 
localized in the radial direction as it is displayed in Figure 9, for instance. The reason for this is qualitatively clear: unstable eigenfunctions should be localized in the region where electric currents are maximal because the current is the reason of instability. If $\alpha>1$, the current is maximal near the outer boundary and, on the contrary, it is maximal near the inner boundary if $\alpha<1$.

As it was pointed out by Spruit (1999), the stabilizing influence of stratification is less pronounced for perturbations with a short radial scale. Therefore, it seems that instability should operate most efficiently on very short radial scales. However, this conclusion is incorrect because the destabilizing effect of electric currents (that is the reason of the Tayler instability) also decreases as the radial scale decreases. It is easy to estimate from the continuity condition, $\nabla \cdot \mathbf{v}=0$, that the radial velocity decreases proportionally to the radial length scale. Therefore, motions tend to be two dimensional (2D) for perturbations with a small radial length scale. However, as shown by Kitchatinov \& Rüdiger (2008), the Tayler instability does not occur in 2D and, hence, it cannot arise for perturbations with very short radial scales although the stabilizing influence of stratification is minimal for them. However, the stabilizing effect of stratification decreases faster as the radial scale decreases and, hence, there should exist some characteristic radial length scale at which the effects of stratification and electric current become comparable. This condition determines a relatively small characteristic width of the eigenfunction.

It was argued by Bonanno \& Urpin (2012) that stability properties of the spherical magnetic configurations are qualitatively different from properties of the cylindrical configurations. For instance, our calculations show that the Tayler instability is most efficient near the equator but the toroidal configurations can be stable near the rotation axis. This conclusion is at variance with the widely accepted opinion (see, e.g., Spruit 1999; Zhang et al. 2003) that the toroidal field is always unstable near the axis. This idea is based on the analysis of cylindrical configurations and on the apparent similarity of the magnetic field topology in the infinite cylinder and the geometry near the rotation axis in spherical geometry. However, there is a qualitative difference between these two cases: the magnetic field in spherical geometry depends generally on the coordinate along the axis and this dependence can provide a stabilizing effect. Therefore, a direct analogy between stability of a cylinder with the azimuthal field and the toroidal field in stellar radiation zones is generally incorrect.

It is well known that stable stratification can suppress the Tayler instability of the toroidal field. Calculations by Bonanno \& Urpin (2012) show that, indeed, the instability does not arise if the Brunt-Väisälä frequency is greater than $\omega_{A 0}$ by a factor of $\sim 5-10$ and the thermal conductivity is neglected. Since $\omega_{\mathrm{BV}}$ is typically high in radiative zones $\left(\sim 10^{-3}\right.$ to $\left.10^{-4} \mathrm{~s}^{-1}\right)$ the instability sets in only if the field is very strong $\left(\geqslant 10^{6}-10^{7} \mathrm{G}\right)$. However, the thermal conductivity reduces the stabilizing effect of stratification drastically. It turns out that the growth rate is non-vanishing for any stratification (Bonanno \& Urpin 2012). Even very strong gravity cannot stop the instability but it only decreases the growth rate. Rotation provides also a strong stabilizing influence on the Tayler instability. The effect of rotation is characterized by the parameter $\eta=2 \Omega / \omega_{A 0}$, which can be large in radiation zones. A reduction of the growth rate becomes significant already at a relatively low angular velocity, $\Omega \sim \omega_{A 0}$, that corresponds to $\eta \sim 1$. All these factors are always present in stellar radiation zones and, therefore, it is of great importance to study the stability of magnetic configurations under their combined influence.

The collective effect of stratification, rotation, and thermal conductivity turns out to be rather unexpected: the instability of the toroidal magnetic field cannot be suppressed if all these three factors are presented except, possibly, in the case of very small values of $\delta$ (which is unrealistic). The combined effect cannot stop the instability but can only decrease the growth rate. The decrease can be substantial, however. For example, a decrease of $\sigma$ caused by stratification and thermal conductivity is inversely proportional to the square of the Brunt-Väisälä frequency. If gravity is strong but the magnetic field is weak, the instability sets in very slowly. Generally, for a sufficiently weak magnetic field, the growth rate can be comparable to the inverse lifetime of a star. Rotation also cannot stop the instability but, such as for stratification and thermal conductivity, it only reduces its growth rate. The growth rate is non-vanishing for any rotation, but it can be drastically reduced in rapidly rotating stars. At large $\eta$, the growth rate decreases $\propto 1 / \eta$. Since the parameter $\eta$ is typically large in the radiation zones, the growth rate should be small. Note, however, that the Alfvén timescale, $\omega_{A 0}^{-1}$, is usually short compared to the lifetime of a star; therefore, even a suppressed instability with a reduced growth rate can play an important role, for instance, in transport processes in radiation zones (mixing, transport of the angular momentum, etc.). It should also be noted that, most likely, the field does not decay to zero because of this instability. When the field becomes weak, the growth rate of the instability is too small. Likely, the field can decay only to the value at which the growth rate becomes comparable to the inverse lifetime of a star.

Note finally that we considered only the ideal case and neglected viscosity and magnetic diffusion. Dissipative effects make the problem much more complicated and we plan to consider them elsewhere. Qualitatively, however, it seems that more or less plausible values of the magnetic diffusivity should not change essentially our results. The point is that the characteristic length scale of the fundamental eigenfunction, despite being relatively short, is still rather large to cause a significant dissipation. Therefore, the magnetic diffusivity should not influence the fundamental eigenfunctions and eigenvalues. The only exception can be very rapidly rotating stars for which the radial scale of fundamental eigenfunctions might be very short. The magnetic diffusivity can also change the properties of modes with large radial numbers and with large $l$ and $m$.

V.U. acknowledges support from the European Science Foundation (ESF) within the framework of the ESF activity "The New Physics of Compact Stars." V.U. also thanks the Russian Academy of Sciences for financial support under the program OFN-15 and INAF-Ossevatorio Astrofisico di Catania for hospitality.

\section{REFERENCES}

Acheson, D. J. 1978, RSPTA, 289, 459

Aleksandrov, A. D., Kolmogorov, A. N., \& Lavrent'ev, M. A. 1963, Mathematics, Its Content, Methods, and Meaning (2nd ed.; Cambridge, MA: MIT Press)

Antia, H. M., Chitre, S. M., \& Thompson, M. J. 2000, A\&A, 360, 335

Balbus, S. A. 1995, ApJ, 453, 380

Bonanno, A., Brandenburg, A., Del Sordo, F., \& Mitra, D. 2012, PhRvE, 86,016313

Bonanno, A., \& Urpin, V. 2008a, A\&A, 488, 1

Bonanno, A., \& Urpin, V. 2008b, A\&A, 477, 35

Bonanno, A., \& Urpin, V. 2011a, PhRvE, 84, 056310 
Bonanno, A., \& Urpin, V. 2011b, A\&A, 525, A100

Bonanno, A., \& Urpin, V. 2012, ApJ, 747, 137

Bonanno, A., Urpin, V., \& Belvedere, G. 2005, A\&A, 440, 199

Bonanno, A., Urpin, V., \& Belvedere, G. 2006, A\&A, 451, 1049

Braithwaite, J. 2006, A\&A, 453, 687

Gilman, P. A. 1970, ApJ, 162, 1019

Godier, S., \& Rozelot, J.-P. 2000, A\&A, 355, 365

Goedbloed, J. P. H., \& Poedts, S. 2004, Principles of Magnetohydrodynamics (Cambridge: Cambridge Univ. Press)

Goossens, M., Biront, D., \& Tayler, R. J. 1981, Ap\&SS, 75, 521

Kitchatinov, L. L. 2008, ARep, 52, 247

Kitchatinov, L., \& Rüdiger, G. 2008, A\&A, 478, 1
Landau, L. D., \& Lifshitz, E. M. 1959, Fluid Mechanics (Course of Theoretical Physics; Oxford: Pergamon)

Markey, P., \& Tayler, R. J. 1973, MNRAS, 163, 77

Mathis, S., \& Zahn, J.-P. 2005, A\&A, 440, 653

Spruit, H. C. 1999, A\&A, 349, 189

Tayler, R. J. 1973, MNRAS, 161, 365

Tayler, R. J. 1980, MNRAS, 191, 151

Urpin, V., \& Gil, J. 2004, A\&A, 415, 305

Urpin, V., \& Rüdiger, G. 2005, A\&A, 437, 23

Velikhov, E. P. 1959, Sov. Phys.-JETP, 9, 995

Zahn, J.-P., Brun, A. S., \& Mathis, S. 2007, A\&A, 474, 145

Zhang, K., Liao, X., \& Schubert, G. 2003, ApJ, 585, 1124 